\title{
TRATAMENTO MATEMÁTICO DOS MODOS VIBRACIONAIS DA MOLÉCULA DE CLOROFÓRMIO
}

\section{MATHEMATICAL TREATMENT OF VIBRATIONAL MODES OF THE CHLOROFORM MOLECULE}

\author{
Maria Helena Rodrigues Mesquita ${ }^{1}$ \\ Adeslaine Negrini Reche ${ }^{1}$ \\ Patrícia Marcondes dos Santos ${ }^{2}$ \\ Yoshiyuki Hase ${ }^{3}$ \\ Mituo Uehara ${ }^{4}$ \\ Kumiko Koibuchi Sakane ${ }^{4}$
}

RESUMO: As frequências dos modos vibracionais da molécula de clorofórmio foram determinadas teoricamente. Os cálculos exigiram um conhecimento de forças interatômicas, comprimentos de ligação e seus ângulos a fim de obter a sua configuração. A molécula pertence ao grupo de ponto $C_{3 v}$ com nove vibrações fundamentais. As coordenadas cartesianas, internas, e de simetria e o campo de força serviram de entrada para um pacote de programas NCT (Tratamento de Coordenadas Normais), para obter frequências vibracionais, distribuição de energia potencial (DEP) e constantes de força de ligação. As atribuições das bandas dos espectros infravermelhos foram feitas baseadas na distribuição de energia potencial calculada.

Palavras-chave: espectroscopia no infravermelho; análise de coordenadas normais; molécula de clorofórmio.

ABSTRACT: The normal vibrational modes of the chloroform molecule were determined theoretically. The calculations required previous knowledge of the force constants, bond lengths, and angles in order to estimate the configuration of chloroform. The molecule belongs to the $C_{3 v}$ point group with nine fundamental vibrational modes. The Cartesian, internal, and symmetry coordinates, along with the force field were used and inputted into the normal coordinate analysis package (NCT) in order to obtain the frequencies, the distribution of the potential energy (DEP), and force constants. The assignment of bands of the infrared spectrum of chloroform was based in the calculated distribution of the potential energy.

Keywords: infrared spectroscopy; normal coordinate analysis; chloroform molecule.

\footnotetext{
1 Mestranda em Bioengenharia - Universidade do Vale do Paraíba - Univap. E-mail: mhrmesquita@hotmail.com lainereche@hotmail.com.

${ }^{2}$ Doutoranda em Engenharia Biomédica - Univap. E-mail: patriciams@univap.br.

${ }^{3}$ Doutor em Química (Físico-Química) - Universidade de São Paulo - USP e Professor da Universidade Estadual de Campinas - Unicamp. E-mail: hase@iqm.unicamp.br.

${ }^{4}$ Doutor(a) em Física - Instituto Tecnológico de Aeronáutica - ITA e Professor(a) Titular da Univap. E-mail: kumiko@univap.br.

* In memoriam.
} 


\section{INTRODUÇÃO}

Nos estudos da espectroscopia vibracional, um dos problemas principais é o melhor entendimento dos modos normais da vibração de molécula e a atribuição dos dados experimentais observados nos espectros infravermelhos.

Uma das técnicas matemáticas mais simples é a análise de coordenadas normais para obter frequências vibracionais fundamentais e suas atribuições por meio da distribuição de energia potencial. Comparando as frequências calculadas e experimentais, pode-se obter os valores de constantes de força de ligação química, fornecendo as indicações das naturezas das ligações químicas e conformações moleculares (COLTHUP; DALY; WIBERLEY, 1990; SALA, 1995).

O método matemático usado para definir estruturas moleculares baseado na análise dos espectros vibracionais exige uma formulação de hipóteses sobre estruturas prováveis. Estas, em geral, são baseadas nos valores experimentais existentes na literatura ou no conhecimento do pesquisador (BARBOSA, 2007; SILVERSTEIN; WEBSTER; KIEMLE, 2007). Antes de recorrer para a qualquer análise matemática, recomenda-se fazer uma tentativa de atribuição das frequências aos modos vibracionais, geralmente, comparando com moléculas semelhantes. $\mathrm{O}$ objetivo deste trabalho é procurar mostrar que a aplicação das propriedades de simetria e da teoria de grupo permite que as atribuições das frequências vibracionais não sejam puramente empíricas, mas que tenham uma base matemática. Para moléculas pequenas, esses tratamentos são indispensáveis para identificar as suas estruturas.
Muitas vezes as moléculas maiores podem ser divididas, hipoteticamente, em várias partes com simetrias localmente definidas, tais com $\mathrm{C}_{2 v}$ ou $\mathrm{C}_{3 v}$. As vibrações características dos grupos $-\mathrm{CH}_{2}$ e $-\mathrm{CH}_{3}$ possuem essas simetrias. Esses fragmentos são analisados, separadamente, como se fossem moléculas menores. Tais análises são, muitas vezes, úteis para caracterizar as frequências vibracionais de biomoléculas (lipídios, proteínas, polissacarídeos, ácidos nucleicos) presentes em sistemas biológicos. Pode-se reconhecer a presença dessas vibrações muitas vezes alteradas e até distorcidas, devido à influência dos fatores externos, tais como algumas toxinas, medicamentos ou alterações nas conformações moleculares (DIEM; GRIFFITHS; CHALMERS, 2008; MANTSCH; CHAPMAN, 1996; STUART, 1997). Variações, na geometria molecular, refletem, geralmente, nas constantes de ligação entre dois átomos. Essas informações estruturais contribuem para o melhor entendimento da complexidade de um sistema biológico.

O presente trabalho consiste em construir um modelo matemático, formado por um conjunto de coordenadas cartesianas, internas, de simetria e campo de força que servem de dados de entrada para um pacote de programas NCT. Estes calculam as frequências vibracionais da molécula e constante de força de ligação química, comparando os valores experimentais e calculados (HASE, 2004).

A molécula de clorofórmio foi escolhida como exemplo para análise vibracional, pois pertence ao grupo de ponto $\mathrm{C}_{3 \mathrm{~V}}$, mesma propriedade de simetria do grupo $-\mathrm{CH}_{3}$, muitas vezes encontrado em biomoléculas. O tratamento de análise de coordenadas normais prevê nove vibrações fundamentais, sendo três modos $A_{1}$ e três duplamente 
degenerados E. (COLTHUP; DALY; WIBERLEY, 1990). E o seu espectro infravermelho possui as bandas definidas que permitem uma boa comparação entre as frequências calculadas e experimentais.

A fórmula estrutural da molécula de clorofórmio é mostrada na Fig. 1.

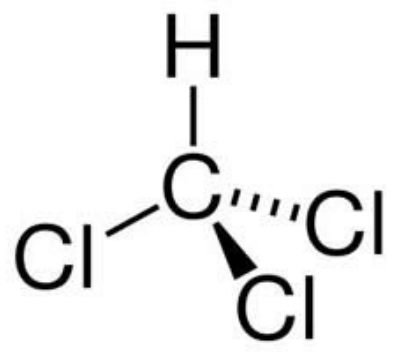

Fig. 1 - Fórmula estrutural da molécula de clorofórmio

Fonte: LOPES (2012).

\section{OBTENÇÃO DOS ESPECTROS INFRAVERMELHOS}

O espectro de clorofórmio no estado líquido foi obtido utilizando cela de cristais de $\mathrm{KBr}$ de um caminho ótico de 0,114 mm, na faixa de 4000 a $600 \mathrm{~cm}^{-1}$, com resolução de $4 \mathrm{~cm}^{-1}$, no modo de transmissão, com 32 varreduras a uma temperatura controlada de $20^{\circ} \mathrm{C}$.

O espectrofotômetro utilizado foi 0 Spectrum GX FT-IR da PerkinElmer. Os espectros foram pré-processados com 0 software Spectrum 5.3 (PerkinElmer), em que foram feitas suavização espectral por meio do algoritmo Savistzky Golay (9 pontos).

A Fig. 2 mostra 0 espectro infravermelho de clorofórmio na região de 3500 a $500 \mathrm{~cm}^{-1}$.

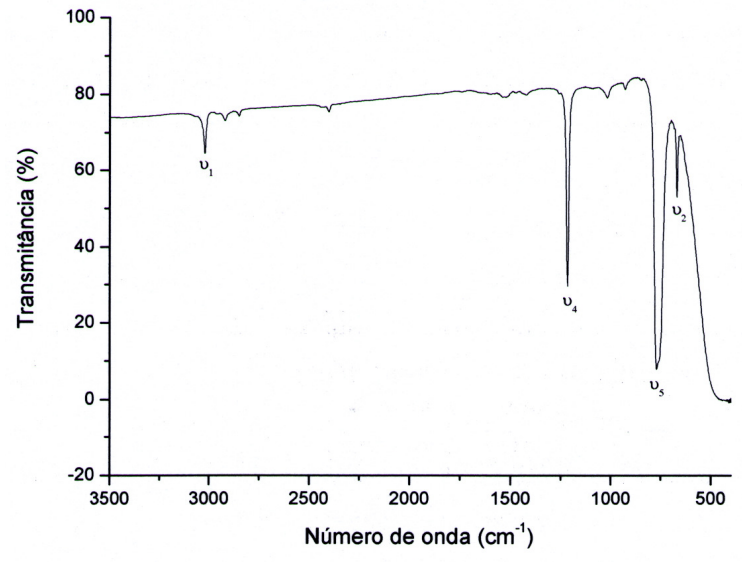

Fig. 2 - Espectro infravermelho de clorofórmio.

\section{ANÁLISE TEÓRICA DAS VIBRAÇÕES DE MOLÉCULAS}

3.1 Determinação de coordenadas internas e construção de coordenadas de simetria

Para este trabalho, o cálculo do número de frequências é feito para estrutura possível da molécula livre do clorofórmio usando os recursos da teoria de grupos de ponto.

A teoria de grupos aplicada às vibrações moleculares acha-se minuciosamente desenvolvida num grande número de livros de espectroscopia (BALL, 2005; COLTHUP; DALY; WIBERLEY, 1990; SALA, 1995).

Aplicamos a simetria $\mathrm{C}_{3 \mathrm{v}}$ para molécula livre do clorofórmio.

Os seguintes valores dos comprimentos e de ângulo de ligação foram utilizados (COLTHUP; DALY; WIBERLEY, 1990): C - H $1.093 \stackrel{0}{\mathrm{~A}}$; C $-\mathrm{Cl} 1.770 \stackrel{0}{\mathrm{~A}}$; $\mathrm{Cl}-\mathrm{C}-\mathrm{Cl}$ $112^{\circ}$.

Os pesos atômicos em u.m.a. foram usados (COLTHUP; DALY; WIBERLEY, 1990): $\mathrm{C}=12.00000 ; \quad \mathrm{H}=1.007825 ; \quad \mathrm{Cl}=$ 35.45300 . 
As coordenadas cartesianas, de todos apresentadas na Tabela 1 (COLTHUP; os átomos constituintes da molécula, são DALY; WIBERLEY, 1990).

Tabela 1 - Coordenadas cartesianas e massas atômicas da molécula de clorofórmio

\begin{tabular}{ccccc}
\hline & $\mathbf{x}$ & $\mathbf{y}$ & $\mathbf{z}$ & Massa atômica \\
\hline $1 . \mathrm{H}$ & 0 & 0 & 1.093 & 1.007825 \\
$2 . \mathrm{C}$ & 0 & 0 & 0 & 12.01115 \\
$3 . \mathrm{Cl}$ & 1.467 & 0.847 & -0.512 & 35.45300 \\
$4 . \mathrm{Cl}$ & 0 & -1.694 & -0.512 & 35.45300 \\
$5 . \mathrm{Cl}$ & 1.467 & 0.847 & -0.512 & 35.45300 \\
\hline
\end{tabular}

As coordenadas internas estão

O número de frequências, em cada apresentadas na Fig. 4 e são:

espécie de simetria, é obtido, para a $R_{1}=r$

molécula de clorofórmio, com a simetria $C_{3 v} \quad R_{2}=t_{1}$

(COLTHUP; DALY; WIBERLEY, 1990). $\quad R_{3}=t_{2}$

$\Gamma_{v}=3 A_{1}+3 E$

$\mathrm{R}_{4}=\mathrm{t}_{3}$

$\mathrm{R}_{5}=\alpha_{1}$

$\mathrm{R}_{6}=\alpha_{2}$

em que $A_{1}$ e $E$ são espécies de simetria.

Portanto, a teoria dos grupos prevê três

modos normais para espécie $A_{1}$ e três

modos degenerados, para espécie $\mathrm{E}$.

A Fig. 3 ilustra nove modos normais para a molécula de clorofórmio.
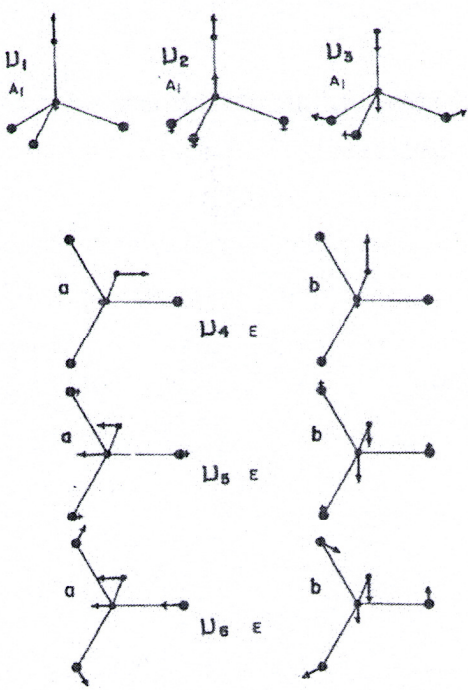

$\mathrm{R}_{7}=\alpha_{3}$

$\mathrm{R}_{8}=\beta_{1}$

$\mathrm{R}_{9}=\beta_{2}$

$R_{10}=\beta_{3}$

$r \quad$ ligação de valência: $\mathrm{C}-\mathrm{H}$

$t_{i} \quad i=1$ até 3 ligação de valência: $\mathrm{C}-\mathrm{Cl}$

$\alpha_{i} \quad i=1$ até 3 ângulo de valência: $\mathrm{Cl}-\mathrm{C}-\mathrm{Cl}$

$\beta_{\mathrm{i}} \quad \mathrm{i}=1$ até 3 ângulo de valência: $\mathrm{H}-\mathrm{C}-\mathrm{Cl}$

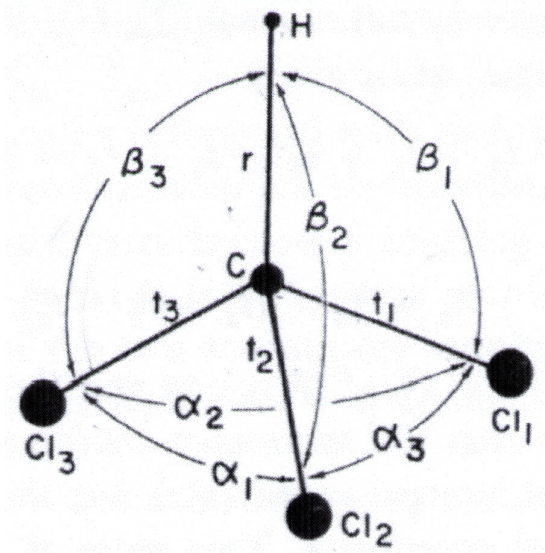

Fig. 4 - Coordenadas internas da molécula de clorofórmio.

Fonte: COLTHUP; DALY; WIBERLEY

(1990).

Fig. 3 - Nove modos normais para a molécula de clorofórmio.

Fonte: COLTHUP; DALY; WIBERLEY

(1990).

A energia potencial de uma molécula é

uma função da distorção da molécula a 
partir da posição de equilíbrio.

Assim, pode-se escrever nossa função potencial com as prováveis interações em coordenadas internas (COLTHUP; DALY; WIBERLEY, 1990).

As constantes de estiramento são:

$$
\begin{array}{ll}
k 1 & k_{r} \\
k 2 & k_{t i} \quad i=1 \text { até } 3
\end{array}
$$

As constantes de deformação angular são:

k3 $\quad \mathrm{k}_{\alpha \mathrm{i}} \mathrm{i}=1$ até 3

k4 $\quad \mathrm{k}_{\beta \mathrm{i}} \mathrm{i}=1$ até 3

As constantes de interação são:

k5

$$
\mathrm{k}_{\mathrm{rti}} \mathrm{i}=1 \text { até } 3
$$

k6

$$
\mathrm{k}_{\text {titj }} \mathrm{i}=1 \text { até } 3, \mathrm{j}=1 \text { até } 3 \text { e } \mathrm{i} \neq \mathrm{j}
$$$$
\mathrm{k}_{\mathrm{r \alpha i}} \mathrm{i}=1 \text { até } 3
$$

k7

k8 $\quad \mathrm{k}_{\mathrm{r} \beta \mathrm{i}} \mathrm{i}=1$ até 3

k9

$$
\mathrm{k}_{\text {tioj }} \mathrm{i}=1 \text { até } 3, \mathrm{j}=1 \text { até } 3 \text { e } \mathrm{i} \neq \mathrm{j}
$$

k10

$$
\mathrm{k}_{\text {tißj }} \quad \mathrm{i}=1 \text { até } 3, \mathrm{j}=1 \text { até } 3 \mathrm{e} \mathrm{i}=\mathrm{j}
$$

$\mathrm{k} 11 \quad \mathrm{k}_{\text {tiaj }} \quad \mathrm{i}=1$ até $3, \mathrm{j}=1$ até $3 \mathrm{e} \mathrm{i}=\mathrm{j}$

k12 $\mathrm{k}_{\mathrm{t} i \beta j} \quad \mathrm{i}=1$ até $3, \mathrm{j}=1$ até 3 e $\mathrm{i} \neq \mathrm{j}$

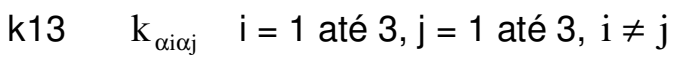

k14 $\mathrm{k}_{\beta i \beta j} \quad \mathrm{i}=1$ até $3, \mathrm{j}=1$ até $3, \mathrm{i} \neq \mathrm{j}$

$\mathrm{k} 15 \quad \mathrm{k}_{\alpha i \beta j} \quad \mathrm{i}=1$ até $3, \mathrm{j}=1$ até $3 \mathrm{e} \mathrm{i}=\mathrm{j}$

k16 $\mathrm{k}_{\text {oißj }} \mathrm{i}=1$ até $3, \mathrm{j}=1$ até $3, \mathrm{i} \neq \mathrm{j}$

As coordenadas de simetria para a molécula de clorofórmio, são apresentadas a seguir: (COLTHUP; DALY; WIBERLEY,
1990).

$$
\text { Espécie de simetria } A_{1}
$$

$$
\mathrm{S}_{1}=\mathrm{R}_{1}
$$

$$
\mathrm{S}_{2}=\frac{1}{\sqrt{3}}\left(\mathrm{R}_{2}+\mathrm{R}_{3}+\mathrm{R}_{4}\right)
$$

$$
S_{3}=\frac{1}{\sqrt{6}}\left(R_{5}+R_{6}+R_{7}-R_{8}-R_{9}-R_{10}\right)(4)
$$

\section{Espécie de simetria $\mathrm{E}$}

$$
\begin{aligned}
& S_{1}=\frac{1}{\sqrt{6}}\left(2 R_{2}-R_{3}-R_{4}\right) \\
& S_{2}=\frac{1}{\sqrt{6}}\left(2 R_{5}-R_{6}-R_{7}\right)
\end{aligned}
$$

$\mathrm{S}_{3}=\frac{1}{\sqrt{6}}\left(2 \mathrm{R}_{8}-\mathrm{R}_{9}-\mathrm{R}_{10}\right)$

$\mathrm{S}_{4}=\frac{1}{\sqrt{2}}\left(\mathrm{R}_{3}-\mathrm{R}_{4}\right)$

$$
\mathrm{S}_{5}=\frac{1}{\sqrt{2}}\left(\mathrm{R}_{6}-\mathrm{R}_{7}\right)
$$

$$
\mathrm{S}_{6}=\frac{1}{\sqrt{2}}\left(\mathrm{R}_{9}-\mathrm{R}_{10}\right)
$$

\subsection{Vibrações moleculares e coordenadas normais}

Uma molécula de clorofórmio pode ser pensada como um conjunto de osciladores acoplados. Faz-se desacoplar esses osciladores em osciladores harmônicos simples, descritos como coordenadas normais com o uso de propriedades de simetria da molécula e da teoria do grupo (COLTHUP; DALY; WIBERLEY, 1990; HASE, 2004; SALA, 1995).

Espera-se uma equação de movimento de nono grau $3 \mathrm{~N}-6=9$ (em que $\mathrm{N}$ 
é igual a nove e representa o número de átomos da molécula de clorofórmio: $\mathrm{CHCl}_{3}$ ) para a molécula em estudo e suas raízes estariam relacionadas às frequências vibracionais. Utilizando propriedades de simetria e teoria de grupo $\mathrm{C}_{3 \mathrm{v}}$, pode-se fatorar as equações em equações de menor grau, mais fáceis de serem resolvidas (COLTHUP; DALY; WIBERLEY, 1990; HASE, 2004; SALA, 1995).

Deve-se efetuar transformações de coordenadas aplicando as operações de simetria do grupo ao qual a molécula pertence para se chegar às coordenadas normais, que descrevem vibrações da molécula. A cada operação é associada uma matriz de transformação e um dos objetivos é determinar essas matrizes (COLTHUP; DALY; WIBERLEY, 1990; HASE, 2004; SALA, 1995).

Em algumas etapas, essas transformações envolvem processos numéricos bastante complexos e o tratamento utilizado é o método de matriz GF de Wilson (WILSON; DECIUS; CROSS, 1955).

A energia cinética da molécula é expressa em coordenadas cartesianas de deslocamento:

$2 \mathrm{~T}=\sum_{\mathrm{i}}\left(\mathrm{m}_{\mathrm{i}} \Delta \dot{\mathrm{x}}_{\mathrm{i}}^{2}+\mathrm{m}_{\mathrm{i}} \Delta \dot{\mathrm{y}}_{\mathrm{i}}^{2}+\mathrm{m}_{\mathrm{i}} \Delta \dot{\mathrm{z}}_{\mathrm{i}}^{2}\right)$

A sua energia potencial é descrita em coordenadas internas que permitirão melhor visualização de campo de forças moleculares:

$2 \mathrm{~V}=\sum_{\mathrm{i}, \mathrm{j}} \mathrm{k}_{\mathrm{i}, \mathrm{j}}\left(\mathrm{R}_{\mathrm{i}} \mathrm{R}_{\mathrm{j}}\right)$

em que m é massa atômica, $\Delta x, \Delta y$ e $\Delta z$ são coordenadas cartesianas de deslocamento, $k$ constante de força de ligação e $R$ é coordenada interna.

A energia cinética é mais facilmente expressa na forma matricial:

$2 \mathrm{~T}=\dot{\mathrm{X}}^{+} \mathrm{M} \dot{\mathrm{X}}$

onde

$\mathrm{M}=\left(\begin{array}{lll}\mathrm{m}_{1} & & \\ & & \\ \mathrm{~m}_{2} & & \\ & \mathrm{~m}_{3} & \\ & & \mathrm{~m}_{4} \\ & & \mathrm{~m}_{5}\end{array}\right)$

Os elementos fora da diagonal são nulos.

Seja $B_{R}$ uma matriz de transformação de coordenadas cartesianas de deslocamento em coordenadas internas:

$\mathrm{R}=\mathrm{B}_{\mathrm{R}} \mathrm{X}$

$\mathrm{X}=\mathrm{B}_{\mathrm{R}}^{-1} \mathrm{R}$

A expressão da energia cinética em coordenadas internas será:

$2 \mathrm{~T}=\mathrm{R}^{+}\left(\mathrm{B}_{\mathrm{R}}^{-1}\right) \mathrm{M}\left(\mathrm{B}_{\mathrm{R}}^{-1}\right) \mathrm{R}$

$2 T=R^{+} G_{R}^{-1} R$

onde

$$
\mathrm{G}_{\mathrm{R}}^{-1}=\left(\mathrm{B}_{\mathrm{R}}^{-1}\right) \mathrm{MB}_{\mathrm{R}}^{-1}
$$

A energia potencial em geral é expressa em coordenadas internas, pois o significado físico das constantes de força se torna imediato.

$2 \mathrm{~V}=\mathrm{R}^{+} \mathrm{F}_{\mathrm{R}} \mathrm{R}$

As coordenadas de simetria são introduzidas para ter o fatoramento das matrizes de energias cinética e potencial.

As coordenadas de simetria 
relacionam-se com as coordenadas internas $S=L_{S} Q$

por matriz de transformação U.

$S=U R$

em que

$$
\mathrm{U}^{-1}=\mathrm{U}^{+}
$$

$$
\text { A expressão da energia cinética em } 2 T=Q^{+} I Q
$$

As expressões de energias cinética e potencial, em termos de coordenadas normais, podem ser escritas:

$$
2 \mathrm{~T}=\mathrm{Q}^{+} \mathrm{L}_{\mathrm{S}}^{+} \mathrm{G}_{\mathrm{S}}^{-1} \mathrm{~L}_{\mathrm{S}} \mathrm{Q}
$$

coordenadas de simetria será escrita por:

$2 \mathrm{~T}=\mathrm{S}^{+} \mathrm{UG}_{\mathrm{R}}^{-1} \mathrm{U}^{+} \mathrm{S}$

$2 \mathrm{~T}=\mathrm{S}^{+} \mathrm{G}_{\mathrm{S}}^{-1} \mathrm{~S}$

em que

$$
\mathrm{G}_{\mathrm{S}}^{-1}=\mathrm{UG}_{\mathrm{R}}^{-1} \mathrm{U}^{+}
$$

em que

$$
\mathrm{F}_{\mathrm{S}}=\mathrm{UF}_{\mathrm{R}} \mathrm{U}^{+}
$$

G e F são as matrizes introduzidas por Wilson.

Como as matrizes já estão fatoradas, temos uma equação secular $|\mathrm{GF}-\mathrm{I} \lambda|=0$ para cada espécie de simetria $A_{1}$ e E. O seu grau é dado pelo número de modos normais para espécies $A_{1}$ e $E$ e as raízes, $\lambda_{i}$ relacionam-se com as frequências vibracionais, $v_{\mathrm{i}}$ pela relação:

$$
\lambda_{\mathrm{i}}=4 \pi^{2} v_{\mathrm{i}}^{2}
$$

As coordenadas normais $\mathrm{e}$ as de simetria relacionam-se por meio da matriz de transformação $L_{s}$ : em que

$$
\begin{aligned}
& \mathrm{L}_{\mathrm{S}}^{+} \mathrm{G}_{\mathrm{S}}^{-1} \mathrm{~L}_{\mathrm{S}}=\mathrm{I} \\
& \mathrm{L}_{\mathrm{S}}^{+}=\mathrm{L}_{\mathrm{S}}^{-1} \mathrm{G}_{\mathrm{S}} \\
& 2 \mathrm{~V}=\mathrm{Q}^{+} \mathrm{L}_{\mathrm{S}}^{+} \mathrm{F}_{\mathrm{S}} \mathrm{L}_{\mathrm{S}} \mathrm{Q} \\
& 2 \mathrm{~V}=\mathrm{Q}^{+} \Lambda \mathrm{Q}
\end{aligned}
$$

em que

$$
\Lambda=\mathrm{L}_{\mathrm{S}}^{+} \mathrm{F}_{\mathrm{S}} \mathrm{L}_{\mathrm{S}}
$$

As energias cinética e potencial podem ser expressas em termos de coordenadas normais $Q_{i}$ :

$$
\begin{aligned}
& 2 \mathrm{~T}=\sum_{\mathrm{i}} \mathrm{Q}_{\mathrm{i}}^{2} \\
& 2 \mathrm{~V}=\sum_{\mathrm{i}} \lambda_{\mathrm{i}} \mathrm{Q}_{\mathrm{i}}^{2}
\end{aligned}
$$

As equações de movimento podem ser obtidas a partir das equações de Lagrange independentemente para cada coordenada $Q_{i}$.

$$
\frac{d}{d t}\left(\frac{\partial T}{\partial Q_{i}}\right)+\frac{\partial V}{\partial Q_{i}}=0 \quad i=1,2,3 \ldots
$$

Os programas chamados BEL, GEL, FEL e NCA, constituintes do pacote NCT (tratamento de coordenadas normais), permitem calcular os elementos das matrizes $B, G^{-1}, F$, finalmente, as frequências vibracionais, distribuição de 
energia potencial e constantes de força de ligação. A energia potencial é escrita em função de coordenadas internas, conforme a equação (12) e qualquer variação das ligações químicas e das deformações angulares refletem na alteração de distribuição de energia potencial da molécula. A percentagem de cada modo vibracional é calculada em relação ao número total de modos presentes em cada banda. O programa NCA fornece esses resultados em percentagem, na forma de DEP (distribuição de energia potencial para cada modo de vibração).

A parte final do programa consiste em refinar os valores de constantes de força, comparando as frequências calculadas e experimentais observadas nos espectros infravermelhos.

\section{RESULTADOS}

As frequências vibracionais observadas, calculadas e DEP são apresentadas na Tabela 2.

\section{Tabela 2 - Números de onda observados, calculados e distribuição de energia potencial}

\begin{tabular}{|c|c|c|c|}
\hline \multicolumn{4}{|c|}{ Espécie de simetria A1 } \\
\hline Numeração & Observados $\left(\mathrm{cm}^{-1}\right)$ & Calculados $\left(\mathrm{cm}^{-1}\right)$ & DEP (\%) \\
\hline$v_{1}$ & 3021.0 & 3021.0 & $v_{C-H}(95 \%)$ \\
\hline$v_{2}$ & 657.0 & 657.0 & $v_{\mathrm{C}-\mathrm{Cl}}(100 \%)$ \\
\hline$v_{3}$ & 366.0 & 366.0 & $\delta_{\mathrm{CCl}_{2}}(11 \%), \delta_{\mathrm{Cl}-\mathrm{C}-\mathrm{H}}(79 \%)$ \\
\hline \multicolumn{4}{|c|}{ Espécie de simetria E } \\
\hline Numeração & Observados $\left(\mathrm{cm}^{-1}\right)$ & Calculados $\left(\mathrm{cm}^{-1}\right)$ & DEP (\%) \\
\hline$v_{4}$ & 1212.0 & 1212.0 & $\delta_{\mathrm{C}-\mathrm{H}}(100 \%)$ \\
\hline$v_{5}$ & 759.0 & 759.0 & $v_{\mathrm{C}-\mathrm{Cl}}(30 \%), \delta_{\mathrm{Cl}-\mathrm{C}-\mathrm{H}}(66 \%)$ \\
\hline$v_{6}$ & 261.0 & 261.0 & $\delta_{\mathrm{CCl}_{2}}(96 \%)$ \\
\hline
\end{tabular}

Nota:

$\begin{array}{ll}\text { DEP } & \text { distribuição de energia potencial } \\ v_{\mathrm{C}-\mathrm{H}} & \text { estiramento da ligação } \mathrm{C}-\mathrm{H} \\ v_{\mathrm{C}-\mathrm{Cl}} & \text { estiramento da ligação } \mathrm{C}-\mathrm{Cl} \\ \delta_{\mathrm{C}-\mathrm{H}} & \text { deformação da ligação } \mathrm{C}-\mathrm{H} \\ \delta_{\mathrm{Cl}-\mathrm{C}-\mathrm{H}} & \text { deformação angular Cl-C-H } \\ \delta_{\mathrm{CCl}_{2}} & \text { deformação angular Cl-C-Cl } \\ v_{4}, v_{5} \text { e } v_{6} & \text { são modos degenerados (espécie E) }\end{array}$

Os modos normais vibracionais, para o clorofórmio, são mostrados na Fig. 3.

Na Tabela 3, são apresentados valores de constantes de força de ligação e as de interação refinadas. 
Tabela 3 - Constantes de força para a molécula de clorofórmio

\begin{tabular}{|c|c|c|}
\hline \multicolumn{3}{|c|}{ Constante de estiramento } \\
\hline Numeração & Constante de estiramento & Valor $(\mathrm{md} / \stackrel{\circ}{\mathrm{A}})$ \\
\hline $\mathrm{k} 1$ & $\mathrm{k}_{\mathrm{r}}$ & 4.795 \\
\hline $\mathrm{k} 2$ & $\mathrm{k}_{\mathrm{ti}}$ & 6.311 \\
\hline \multicolumn{3}{|c|}{ Constante de deformação } \\
\hline Numeração & Constante de deformação & Valor $(\mathrm{md} / \stackrel{\circ}{\mathrm{A}})$ \\
\hline k3 & $\mathrm{k}_{\alpha \mathrm{i}}$ & 1.316 \\
\hline k4 & $\mathrm{k}_{\beta \mathrm{i}}$ & 0.265 \\
\hline \multicolumn{3}{|c|}{ Constante de interação } \\
\hline Numeração & Constante de interação & Valor $(\mathrm{md} / \stackrel{\circ}{\mathrm{A}})$ \\
\hline k5 & $\mathrm{k}_{\mathrm{rti}}$ & -1.099 \\
\hline k6 & $\mathrm{k}_{\mathrm{titj}}$ & -0.046 \\
\hline k7 & $\mathrm{k}_{\mathrm{r \alpha i}}$ & 0.293 \\
\hline k8 & $\mathrm{k}_{\mathrm{r} \beta \mathrm{i}}$ & 0.300 \\
\hline k9 & $\mathrm{k}_{\mathrm{ti \alpha j}}$ & 0.357 \\
\hline $\mathrm{k} 10$ & $\mathrm{k}_{\mathrm{ti \beta j}}$ & 0.151 \\
\hline $\mathrm{k} 11$ & $\mathrm{k}_{\mathrm{ti \alpha j}}$ & -0.101 \\
\hline $\mathrm{k} 12$ & $\mathrm{k}_{\mathrm{ti \beta j}}$ & -0.108 \\
\hline k13 & $\mathrm{k}_{\alpha i \alpha j}$ & 0.240 \\
\hline k14 & $\mathrm{k}_{\beta i \beta j}$ & 0.004 \\
\hline k15 & $\mathrm{k}_{\alpha i \beta j}$ & -0.020 \\
\hline $\mathrm{k} 16$ & $\mathrm{k}_{\alpha i \beta j}$ & 0.050 \\
\hline
\end{tabular}

Os termos $\mathrm{k}_{\mathrm{r}}$ e $\mathrm{k}_{\mathrm{ti}}$ indicam variação nos comprimentos de ligação $r$ e $t$ respectivamente.

Os termos $\mathrm{k}_{\alpha \mathrm{i}}$ e $\mathrm{k}_{\beta \mathrm{i}}$ indicam variações de ângulos ai e $\beta \mathrm{i}$, respectivamente.

Os termos $\mathrm{k}_{\mathrm{rti}}, \mathrm{k}_{\mathrm{r} \beta \mathrm{i}}$ e $\mathrm{k}_{\alpha i \alpha j}$ indicam interações entre $r$ e ti; $r$ e $\beta i$; $\alpha i$ e $\alpha$, respectivamente. Observamos que os valores de constantes de estiramento são sempre maiores em relação aos outros.
Muitas vezes, as constantes de interação não são disponíveis e somente as constantes de interação das frequências próximas dentro da matriz da função potencial são consideradas.

As frequências calculadas a partir das constantes de força encontradas na literatura (COLTHUP; DALY; WIBERLEY, 1990) foram comparadas às experimentais, ajustando valores de constantes de força de ligação. Os cálculos são repetidos até que os valores calculados se tornem iguais aos experimen-tais. As constantes de força, assim obtidas, foram apresentadas na Tabela 2. 
Os resultados de distribuição de energia potencial mostram que os modos, 3021; 1217; 657 e $261 \mathrm{~cm}^{-1}$ são praticamente genuínos. No entanto, os modos em 759 e $366 \mathrm{~cm}^{-1}$ representam uma mistura de vários modos de vibração.

\section{CONCLUSÃO}

Os espectros infravermelhos em geral trazem informações completas da estrutura da molécula. No entanto, muitos efeitos surgem ao mesmo tempo; por exemplo: o aspecto das bandas devido à alteração na distribuição eletrônica, à mudança na conformação, e ao acoplamento dos modos, entre outros.

O tratamento matemático das coordenadas normais, para o clorofórmio com a simetria $\mathrm{C}_{3 v}$, foi feito com o uso do pacote de programas chamado NCT.

Muitas vezes, as atribuições das frequências dos espectros infravermelhos são feitas a partir dos compostos semelhantes que existem na literatura. Neste trabalho, as atribuições de frequências foram feitas baseadas na distribuição de energia potencial que é uma função da distorção de suas coordenadas internas. Os resultados mostraram que as bandas em 3021; 1217; 657 e $261 \mathrm{~cm}^{-1}$ representam, praticamente, modos genuínos ao passo que aquelas em 759 e $356 \mathrm{~cm}^{-1}$, uma mistura de vários modos de vibrações.

\section{AGRADECIMENTOS}

Patrícia Marcondes dos Santos agradece a bolsa de estudo concedida pela Fundação Valeparaibana de Ensino - FVE.

\section{REFERÊNCIAS BIBLIOGRÁFICAS}

BALL, D. W. Físico-Química. São Paulo: Thomson, 2005.

BARBOSA, L. C. A. Espectroscopia no Infravermelho na caracterização de compostos orgânicos. Viçosa: UFV, 2007.

COLTHUP, N. B.; DALY, L. H.; WIBERLEY, S. E. Introduction to Infrared and Raman Spectroscopy. 3.ed. New York: Academic Press, 1997.

DIEM, M., GRIFFITHS, P. R.; CHALMERS, J. M. Vibrational Spectroscopy for Medical Diagnosis. Chichester: Wiley, 2008.

HASE, Y. NCT: Pacote de Tratamento de Coordenadas Normais (Versão 7). Química Nova, v. 27, n. 4, p.664-67, 2004.

LOPES, P. Clorofórmio. Brasil Escola. Disponível em: http://www.brasilescola.com/drogas/clorofor mio.htm. Acesso em: 13 jun. 2012.

MANTSCH, H. H.; CHAPMAN, D. Infrared Spectroscopy of Biomolecules. New York: Wiley-Liss, 1996.

SALA, O. Fundamentos da espectroscopia Raman e no Infravermelho. São Paulo: Editora UNESP, 1995.

SILVERSTEIN, R. M.; WEBSTER, F. X.; KIEMLE, D. J. Identificação espectrométrica de compostos orgânicos. 7. ed. Rio de janeiro: LTC, 2007.

STUART, B. H. Biological Applications of Infrared Spectroscopy. Chichester, New Jork, John Wiley \& Sons, 1997.

WILSON, E. B.; DECIUS, J. C.; CROSS, P. Molecular Vibrations. New York: McGrawHill, 1955. 